\title{
过渡金属催化合成胍类衍生物研究进展
}

\author{
徐树英 ${ }^{a}$ 陈小佳 ${ }^{b}$ 黎吉辉*, ${ }^{*}$ 许文茸 ${ }^{a}$ 张玉苍 ${ }^{*, a}$ \\ ( ${ }^{a}$ 海南大学材料与化工学院 海口 570228) \\ ( ${ }^{b}$ 海南省产品质量监督检验所 海口 570003)
}

\begin{abstract}
摘要 胍 $\left(\mathrm{R}^{1} \mathrm{NHC}\left(=\mathrm{NR}^{2}\right) \mathrm{NHR}^{3}\right)$ 为一类独特的三氮化合物, 其结构单元广泛存在于天然产物和药物分子中. 他们具有 多样性生物活性, 在药物和农药领域都有广泛应用; 此外, 其在有机合成和功能材料领域也有很高的应用价值. 由于 胍独特的结构和广泛的应用价值, 其合成方法的研究已成为有机合成研究的一个热点. 近年来不断有胍的新合成方法 涌现, 特别是高效的过渡金属催化合成方法. 本文主要对近 20 年来发展的过渡金属催化构筑 $\mathrm{C}-\mathrm{N}$ 键反应合成胍类化 合物的方法进行综述，全面介绍各类胍化合物合成方法的研究现状，总结归纳不同过渡金属催化合成方法的规律、特 点及不足, 为过渡金属催化合成胍类化合物的研究提供帮助.
\end{abstract}

关键词 过渡金属催化; $\mathrm{C}-\mathrm{N}$ 键生成反应; 胍类衍生物; 合成方法

\section{Recent Advances in Transition Metal-Catalyzed Synthesis of Guanidines}

\author{
$\mathrm{Xu}_{\text {, Shuying }}{ }^{a} \quad$ Chen, Xiaojia $^{b} \quad \mathrm{Li}$, Jihui ${ }^{*, a} \quad \mathrm{Xu}$, Wenrong $^{a} \quad$ Zhang, Yucang $^{*, a}$ \\ $\left({ }^{a}\right.$ College of Materials and Chemical Engineering, Hainan University, Haikou 570228) \\ ( ${ }^{b}$ Products Quality Supervision and Testing Institute of Hainan Province, Haikou 570003)
}

\begin{abstract}
Guanidines are a unique class of tri-nitrogen compounds having various bioactivities, which are wildly presented in natural products and drug molecules, and they are wildly used in pharmacy and pesticide fields. Moreover, they have high application value in organic synthesis and functional materials. The synthesis of guanidines has received much attention due to their unique structures and widespread application value. Recently, lots of new synthetic methods of guanidines, especially the synthetic methods based on highly efficient transition metal-catalyzed reactions, have been developed. The recent progress in transition metal-catalyzed reactions developed for the synthesis of guanidines in the past twenty years is summarized. The synthetic methods for various guanidines are introduced comprehensively, and the characteristics and disadvantages of these methods are also discussed, thus it may be helpful for the research on transition metal-catalyzed synthesis of guanidines.
\end{abstract}

Keywords transition metal-catalyzed; $\mathrm{C}-\mathrm{N}$ formation reaction; guanidines; synthetic method

胍 $\left[\mathrm{R}^{1} \mathrm{NHC}\left(=\mathrm{NR}^{2}\right) \mathrm{NHR}^{3}\right]$ 为一类重要的强碱性三氮 化合物. 如天然精氨酸为生物体内常见的胍类化合物, 具有重要的生理功能 ${ }^{[1]}$. 此外, 胍单元广泛存在于天然 产物及人工合成药物分子中 ${ }^{[2]}$. 胍独特的三氮骨架及显 著的生物活性使其在药物、农药、有机合成、材料等领 域都有广泛的应用. 如双胍类化合物可用于治疗糖尿 病, 代表性的化合物有山羊豆碱、二甲双胍、苯乙双胍、 丁双胍等, 二甲双胍还可以用于治疗多囊卵巢综合症.
此外，在有机合成中胍常被广泛用作有机中间体、有机 小分子催化剂和过渡金属配体 ${ }^{[3]}$, 最近还有报道证实锞 系胍类络合物具有催化醛二聚反应的活性 ${ }^{[4]}$.

胍类化合物在不同的领域都有广泛的应用，对其合 成方法的研究已成为有机合成研究的热点. 自 1981 年 通过人工合成第一个胍类化合物以来, 经过 100 多年的 研究, 合成化学家们已发展了大量的胍类化合物合成方 法. 早期胍类化合物合成方法主要基于经典的亲核取代

\footnotetext{
*E-mail: lijihui@hainu.edu.cn, zhangyucang88@163.com

Received April 18, 2016; revised May 16, 2016; published online June 20, 2016.

Project supported by the Natural Science Fundation of Hainan Province (Nos. 20162015, 20152027), the Hainan Province International Science and Technology Specific (No. KJHZ2014-02) and the Research Fund for the Doctoral Program of Higher Education of China (No. 20134601110004).

海南省自然科学基金(Nos. 20162015, 20152027)、海南省国际科技合作专项(No. KJHZ2014-02)、教育部高等学校博士学科点专项科研基金(No. 20134601110004)资助项目.
} 
和亲核加成反应 ${ }^{[5]}$. 伴随着胍在农业、医药、化工及材 料等领域应用的推广，对胍类化合物的合成要求也不断 提高, 促进了胍类化合物合成方法的发展. 近年来, 涌 现出大量过渡金属催化反应合成胍类化合物的新方法.

过渡金属作为一类高效、多功能的催化剂, 其催化 的反应可简便构建 $\mathrm{C}-\mathrm{C}$ 键 $^{\left[{ }^{6]}\right.} 、 \mathrm{C}-\mathrm{N}$ 键 ${ }^{[7]} 、 \mathrm{C}-\mathrm{O}$ 键 ${ }^{[8]}$ 、

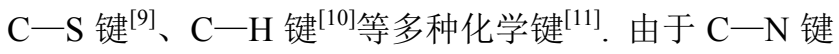
广泛分布在医药中间体、农药中间体、有机材料、天然 产物等有机化合物中, 因此过渡金属催化构建 $\mathrm{C}-\mathrm{N}$ 键 反应是近年来现代机合成化学研究热点之一. 研究发现 过渡金属催化构建 $\mathrm{C}-\mathrm{N}$ 键反应可简便高效地合成各种 胍类化合物, 包括链状胍和环状胍.

通过经典的亲核取代反应和亲核加成反应合成链 状胍类化合物的综述已有相关报道 ${ }^{[5]}$, 故本文主要综述 近 20 年来(1996 年来)发展的过渡金属催化合成胍方法 学研究. 根据合成胍类化合物反应的特点可分为胍类化 合物的反应和生成胍官能团的反应两种.

\section{1 胍类化合物的反应}

过渡金属催化胍的反应为一类直接合成胍类化合 物的有效方法, 可简便合成多种链状和环状胍类化合 物.

\section{1 链状胍类化合物的合成}

链状胍类化合物的常见过渡金属催化合成方法主 要基于链状胍和其它试剂的 $\mathrm{C}-\mathrm{N}$ 偶联反应，反应可以 简便地对链状胍进行修饰或者合成更复杂的链状胍类 化合物.

2010 年, Antilla 小组 ${ }^{[12]}$ 报道了铜催化简单易得的胍 盐酸盐(1)和芳基碘 2 的 Ullmann 偶联反应合成对称的 $N, N^{\prime}$-双芳基胍 3 (Eq. 1); 利用 $N, N^{\prime}$-二乙基水杨酰胺 (L-1) 作为配体和 $\mathrm{K}_{3} \mathrm{PO}_{4}$ 作为碱, 在 $\mathrm{CuI}$ 催化下胍盐和芳 基碘化合物即可平稳地反应生成 $N, N^{\prime}$-双芳基胍产物. 多种芳基碘代物都适用于该反应体系，但反应产率因底 物的不同有较大的波动 $(19 \% \sim 92 \%)$, 普遍较低, 且反 应只适合于对称的 $N, N^{\prime}$-双芳基胍的合成. 后来, $\mathrm{Ma}$ 课 题组 ${ }^{[13]}$ 又发展了高效的 $\mathrm{CuI} / N$-methylglycin 催化体系. 相较于 Antilla 小组的催化体系, Ma 小组发展的催化体 系可以同时兼容多种芳基碘和芳基溴底物, 且催化活性 较高，得到中等偏高的产率( $49 \% \sim 90 \%)$. 此外，该催化 体系除了可以催化合成对称的 $N, N^{\prime}$ 二二芳基胍, 还可以 催化合成不对称的 $N, N^{\prime}$ 二二芳基胍. 不对称胍的合成通 过两步一锅者反应实现, 胍硝酸盐先和贫电子的芳基碘 代物进行单芳基化反应，再和另一种芳基碘化物反应一 锅合成不对称的 $N, N^{\prime}$-双芳基胍.

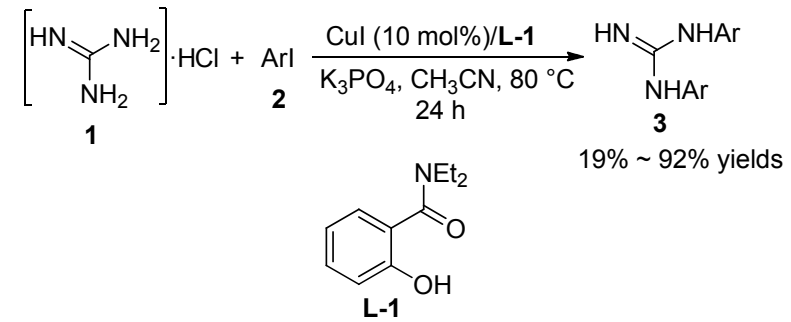

钯或者铱催化胍分子的烯丙基化反应可以对胍进 行官能化合成烷基取代链状胍类化合物 (Scheme 1). Takemoto 和其合作者 ${ }^{[14]}$ 报道了 $\mathrm{Pd}\left(\mathrm{PPh}_{3}\right)_{4}$ 或者 $[\mathrm{IrCl}(\mathrm{COD})]_{2}$ 催化烯丙醇酯 $\mathbf{5}$ 和胍 $\mathbf{4}$ 的烯丙基化反应，实 现了对胍类化合物的单或者双烯丙基官能化. 有趣的是 这两种催化剂表现出了不同的选择性, $\mathrm{Pd}\left(\mathrm{PPh}_{3}\right)_{4}$ 催化反 应生成的是直链产物 6 或者 7, 而 $[\mathrm{IrCl}(\mathrm{COD})]_{2}$ 催化反应 生成的是支链产物 8 或者 9 . 对于单芳基化反应，不对 称胍的反应往往生成两种产物(Scheme 1, a). 此外，作 者将配体 pybox 和 $[\mathrm{IrCl}(\mathrm{COD})]_{2}$ 组合成不对称催化体系, 催化胍和烯丙基亚磷酸酯的偶联反应实现了对一些手 性胍类化合物的不对称合成(Scheme 1, b). 该反应可简 便合成直链和支链烯丙基胍类化合物; 但底物适用范围 窄，需要多个拉电子基团活化胍单元.

\section{2 环状胍类化合物的合成}

2-氨基苯并咪唑又称 $N, N^{\prime}$-邻亚苯基胍，是一类很 重要的环状胍类化合物, 具有多样的生理活性, 如杀 菌、抗菌、抗炎、利尿、降血压等活性，在农药和医药 中都有广泛的应用 ${ }^{[15]}$. 另外，其在有机合成中也是一类 重要的有机中间体和有机催化剂 ${ }^{[16]}$. 早期, 2-氨基苯并 咪唑的合成主要是通过邻苯二胺类化合物的反应来实 现 ${ }^{[17]}$. 伴随着 2-氨基苯并咪唑类化合物在不同领域的应 用，近年来 2-氨基苯并咪唑类化合物的合成引起了合成 化学家的广泛关注, 发展了各种过渡金属催化合成方 法，下面将对其进行详细的介绍.

铜催化胍和芳基卤代物的分子间 $\mathrm{C}-\mathrm{N}$ 偶联反应可 以合成链状胍类化合物, 而邻卤代芳基胍分子内的 $\mathrm{C}-$ $\mathrm{N}$ 偶联反应则可以实现对环状胍类化合物 2-氨基苯并咪 唑的合成. 2003 年 Batey 等 ${ }^{[18]}$ 以 $\mathrm{CuI} / 1,10$-Phen 或者是 $\operatorname{Pd}\left(\mathrm{PPh}_{3}\right)_{4}$ 为催化剂, 催化 1-(2-卤代芳基)-胍(10)在碱性 条件下进行分子内 $\mathrm{C}-\mathrm{N}$ 偶联反应合成 2-氨基苯并咪唑 化合物 11 (Scheme 2,a). 研究发现 CuI/1,10-Phen 催化体 系的产率普遍比 $\mathrm{Pd}\left(\mathrm{PPh}_{3}\right)_{4}$ 催化体系的高; 此外, 对于含 两种不同 $\mathrm{N}-\mathrm{H}$ 键的胍, 这两种过渡金属催化的反应表 现了不同的区域选择性. 最近 Xi 课题组 ${ }^{[19]}$ 以醋酸碘苯 或者是三氟醋酸碘苯为促进剂，在无过渡金属条件下， 
(a) Palladium- and iridium-catalyzed allylation:

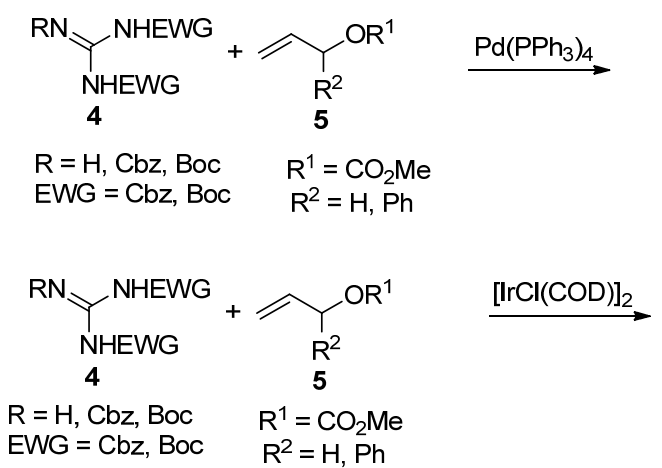

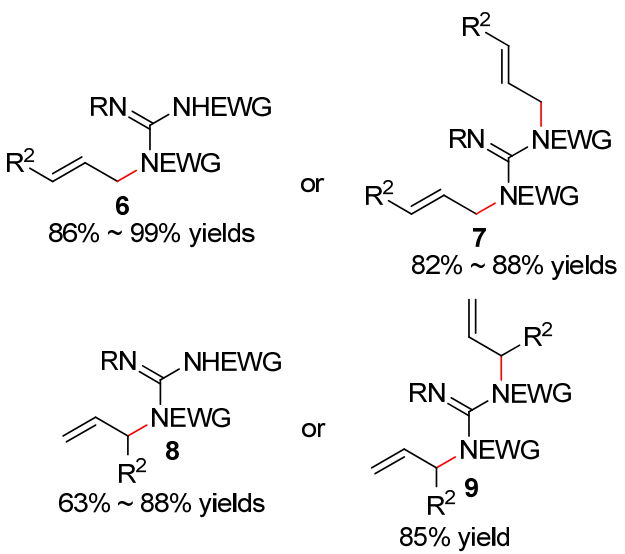

(b) Iridium-catalyzed asymmetric allylation:

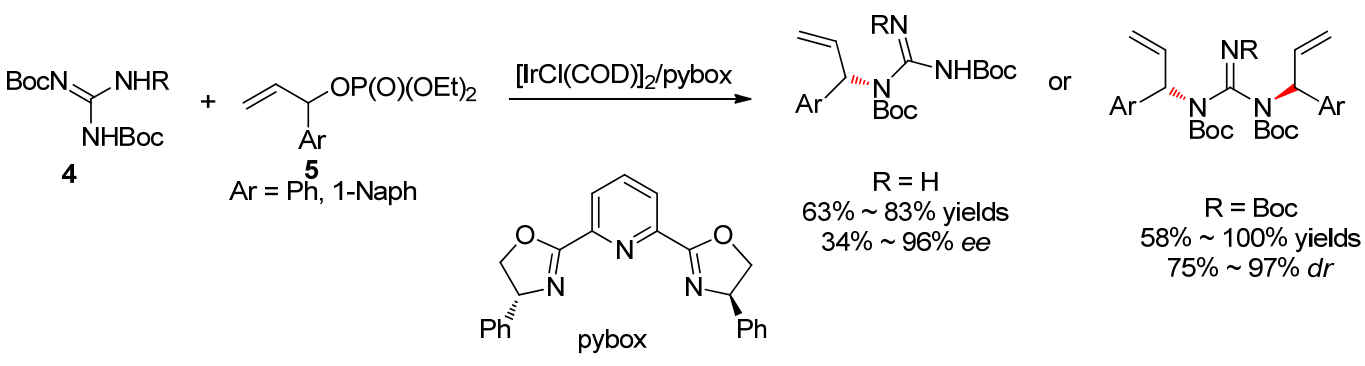

图式 1 钯和铱催化胍烯丙基化反应.

Scheme 1 Palladium and iridium-catalyzed allylation of guanindines

(a) Copper- or palladium-catalyzed synthesis of 2-aminobenzimidazoles:

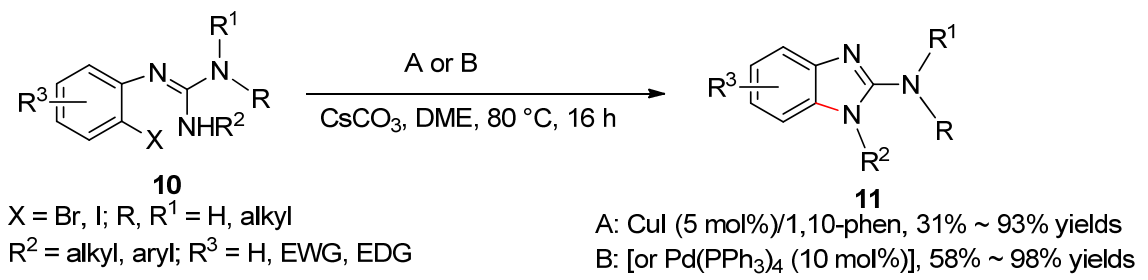

(b) $\mathrm{Phl}(\mathrm{OAc})_{2}$ or $\mathrm{Phl}(\mathrm{TFA})_{2}$ promoted synthesis of 2-aminobenzimidazoles:

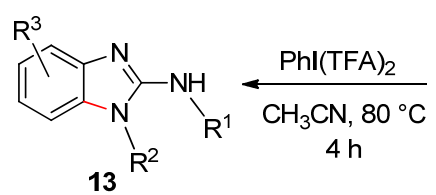

$32 \% \sim 83 \%$ yields

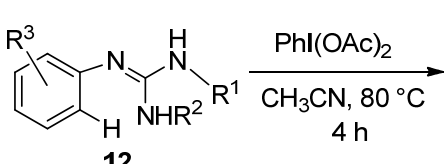

12

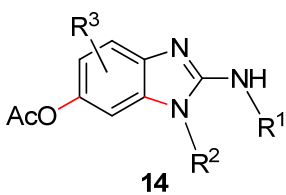

$38 \% \sim 75 \%$ yields

图式 2 分子内 $\mathrm{C}-\mathrm{N}$ 偶联合成 2-氨基苯并咪唑.

Scheme 2 Intramolecular $\mathrm{C}-\mathrm{N}$ coupling for synthesis of 2-aminobenzimidazoles

也可以实现胍类化合物 12 的分子间 $\mathrm{C}-\mathrm{N}$ 氧化偶联反 应, 构建 2-氨基苯并咪唑(Scheme 2, b). 以醋酸碘苯为 促进剂, 反应生成的产物为 6-乙酰氧基-2-氨基苯并咪 唑; 而以三氟醋酸碘苯为促进剂, 产物为 2-氨基苯并咪 唑. 该方法原料比较简单, $N$-芳基胍的芳基邻位不需要 官能化, 且该反应可以避免使用过渡金属催化剂, 这些 都是该方法的优势.

2-氨基苯并咪唑的直接 $N$-芳基化也可以用于合成
多样性的 2-氨基苯并咪唑类化合物. Buchwald 小组 ${ }^{[20]}$ 利用 $\mathrm{CuI}$ 和 $\mathrm{Pd}_{2}(\mathrm{dba})_{3}$ 催化 2-氨基苯并咪唑 15 和卤代芳 烃 16 的偶联反应合成 2-氨基苯并咪唑衍生物(Scheme 3). 该方法的优势为: 通过使用不同的催化剂可以实现 对 $N$-芳基化选择性控制，以 $\mathrm{CuI}$ 为催化剂反应发生在咪 唑环的 $\mathrm{N}$ 上, 而以 $\mathrm{Pd}_{2}(\mathrm{dba})_{3}$ 为催化剂反应在 2 位氨基上 进行. 反应可简便合成多种 2-氨基苯并咪唑, 得到很高 的产率. 随着胍类化合物合成研究的深入，其研究对象 
已拓展至广泛存在于天然产物和人工合成活性分子中 的脂肪胍环.

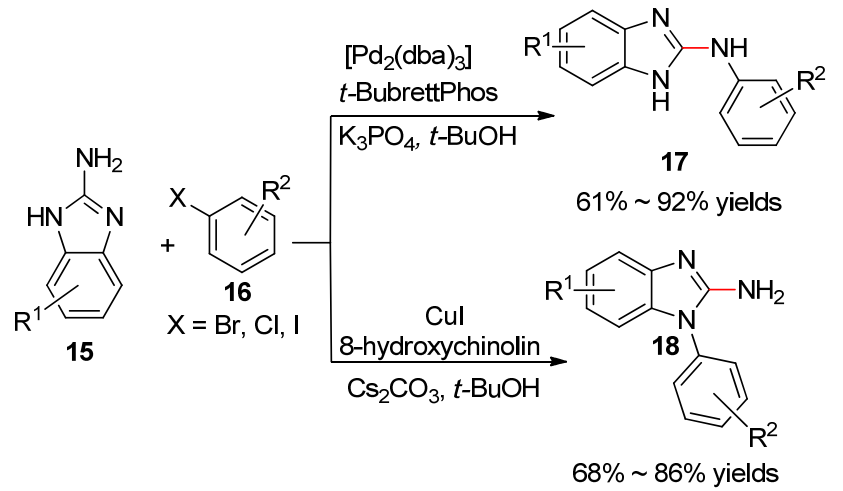

图式 3 催化剂控制 $\mathrm{C}-\mathrm{N}$ 偶联反应选择性合成 2-氨基苯并咪 唑化合物

Scheme 3 Catalysts-controlled $\mathrm{C}-\mathrm{N}$ coupling for selective synthesis of 2-aminobenzimidazoles

铑可以和氨基化合物反应生成高活性的铑氮卡宾 中间体，该中间体被广泛应用于氨基化反应和氮环丙烷 化反应中构建 $\mathrm{C}-\mathrm{N}$ 键. 最近 Bois 和其合作者 ${ }^{[21]}$ 发现铑 可以催化烷基胍的分子内氮卡宾 $\mathrm{C}-\mathrm{H}$ 插入反应, 实现 $\mathrm{sp}^{3}-\mathrm{C}-\mathrm{H}$ 键的选择性活化构建五元胍环. 以 $\mathrm{Rh}_{2}(\mathrm{esp})_{2} /$ $\mathrm{PhI}(\mathrm{OAc})_{2}$ 催化胍 19 的分子内氨基化反应合成五元脂肪 胍环 $20(\mathrm{Eq} .2)$. 在 $\mathrm{PhI}(\mathrm{OAc})_{2}$ 的氧化作用下, 胍和 $\mathrm{Rh}_{2}(\mathrm{esp})_{2}$ 反应先生成铑的氮卡宾中间体，接着 $\mathrm{N}$ 烷基的 $\beta-\mathrm{sp}^{3}-\mathrm{C}-\mathrm{H}$ 键插入到铑的氮卡宾生成新的 $\mathrm{C}-\mathrm{N}$ 键形成 五元胍环. 该反应可以获得很好的区域选择性和产率; 同时反应条件温和，可以兼容烯烃、酯及吲哚，因此该 反应可能有潜在的应用.

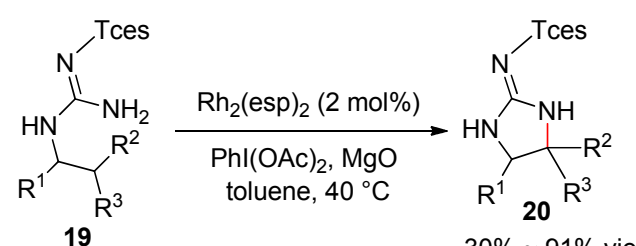

$\mathrm{R}^{1}=\mathrm{H}$, COOBu- $t ; \mathrm{R}^{2}, \mathrm{R}^{3}=\mathrm{H}$, alkyl, aryl Tces $=(2,2,2$-trichloroethoxy)sulfonyl

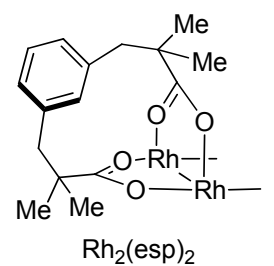

过渡金属催化不饱和 $\mathrm{C}-\mathrm{C}$ 键的氢胺化反应为一类 高效简洁构建 $\mathrm{C}-\mathrm{N}$ 键的合成方法, 分子内胺基化反应 已被证实是一类高效构建氮杂环化合物的合成方法 ${ }^{[22]}$, 反应中无任何副产物生成，具有 $100 \%$ 的原子经济性.
过渡金属催化氢胺化可构建胍环化合物, Looper、Muñiz 和 Wolfe 课题组 ${ }^{[23]}$ 在这方面做了系统的研究.

2011 年 Looper 等 ${ }^{[24]}$ 发展了过渡金属催化炔丙基胍 21 氢胺化反应，他们发现利用不同的过渡金属可以实 现对分子内氢胺化反应的区域选择性控制(Scheme 4). 以 $\mathrm{AgOAc}$ 为催化剂, 反应主要发生 5-外型-对角系环加 成生成五元胍环 22 , 而以 $\mathrm{Rh}(\mathrm{oct})_{4}$ 为催化剂则主要进行 6-内型对角系环加成反应产生六元胍类化合物 23. 该反 应条件较温和，能够兼容烯烃活性官能团. 含有烯丙基 的炔丙基胍的反应选择性发生在参建上，而双键不受影 响; 该产物有构建天然胍类化合物环骨架的潜在应用. 反应无任何副产物生产，具有 $100 \%$ 的原子经济性.

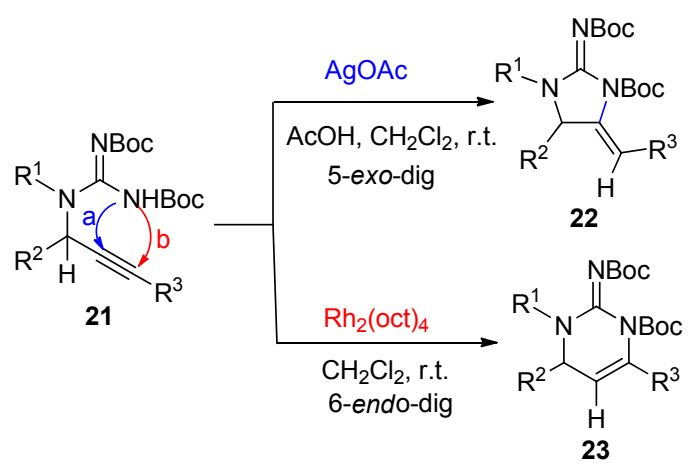

图式 4 过渡金属催化剂控制分子内氢胺化选择性合成五元 和六元胍

Scheme 4 Transition-metal catalyst-controlled selective intramolecular hydroamination for the synthesis of 5- and 6-membered cyclic guanidines

Muñiz 等 ${ }^{[25]}$ 证实过渡金属催化含双键胍类化合物 的分子内双胺基化反应可制备稠环胍类化合物. 他们以 $\mathrm{Pd}(\mathrm{OAc})_{2} / \mathrm{CuCl}_{2}$ 共同促进含双键胍 24 的分子内双氨基 化合成稠环脂肪胍 25 (Scheme 5). 在钯的作用下，胍 24

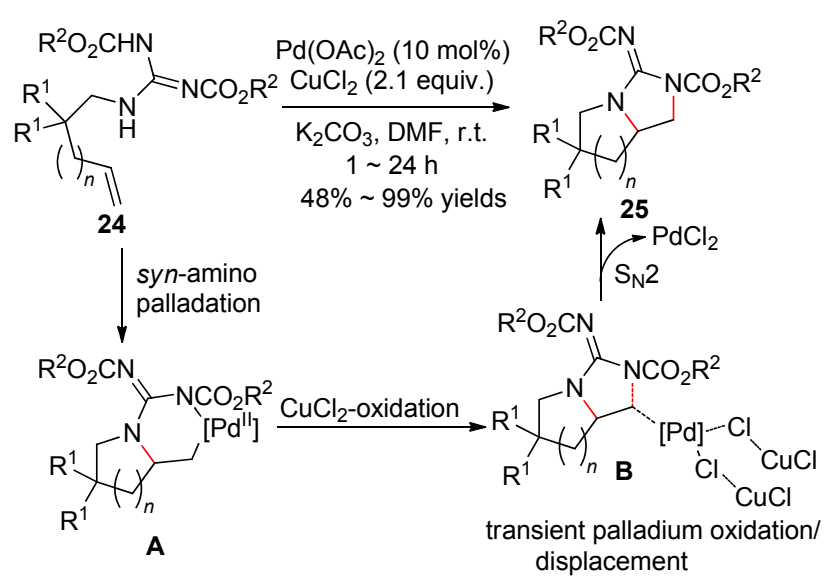

图式 5 钯催化胍分子内双氨基化反应

Scheme 5 Palladium-catalyzed intramolecular diamination of guanidines 
的双键发生顺式氨基钯化反应生成中间体 $\mathbf{A}$, 接着在 $\mathrm{CuCl}_{2}$ 促进下进行氧化及随后的分子内亲核取代钯反应 生成二环胍和二价钯, 然后开始新的催化循环. 和上述 胍分子内反应相比, 该反应的优点为一步构建两个 C$\mathrm{N}$ 键, 是一类高效和高原子经济性的合成方法.

基于不饱和双键的氢胺化反应, 2013 年 Wolfe 课题 组 ${ }^{[26]}$ 发展了钯催化多米诺反应构建多取代五元胍环的 新方法. 他们以 $\mathrm{Pd}_{2}(\mathrm{dba})_{2} / \mathrm{Nixantphos}$ 为催化体系, 催化 烯丙基胍 26 和卤代烃 27 的多米诺反应合成五元脂肪胍 环类化合物 28 (Eq. 3). 通过利用不同的卤代烃即可简 便高效地合成一系列五元环状胍类化合物, 反应一步可 以构建一个 $\mathrm{C}-\mathrm{N}$ 键和一个 $\mathrm{C}-\mathrm{C}$ 键, 能很好地兼容酮 基、缩醛及烯基等官能团. 基于实验结果，作者提出了 以下反应机理: 钯和卤代物首先进行氧化加成, 接着和 胍在碱作用下反应生成氨基钯中间体 IM-1, IM-1 进行 $\mathrm{C}-\mathrm{C}$ 双键迁移插入 $\mathrm{N}-\mathrm{Pd}$ 键, 后续的还原消除反应即 可生成五元胍环.

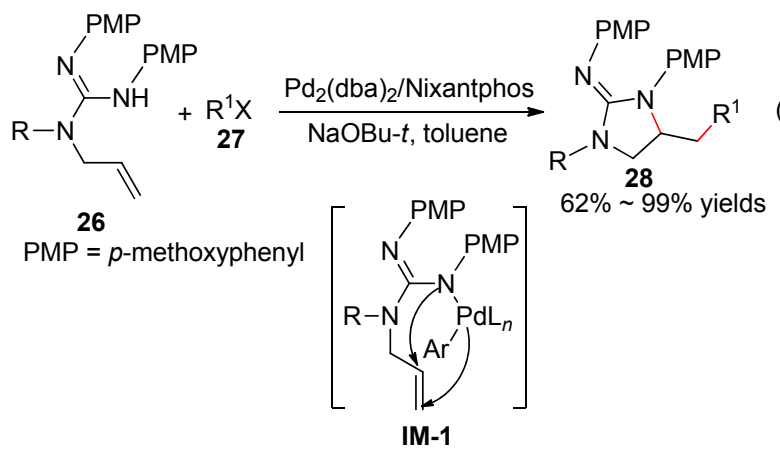

Wolfe 课题组 ${ }^{[27]}$ 又发展了 $\mathrm{Pd}(\mathrm{OAc})_{2} /$ Ruphos 催化炔 丙基胍 29 和芳基三氟甲磺酸酯 $\mathbf{3 0}$ 的氨基化/芳基化多米 诺反应合成 2-氨基咪唑类化合物 $\mathbf{3 1}$ (Scheme 6). 反应条 件能够很好地兼容氢胺化和 $\mathrm{C}-\mathrm{C}$ 偶联反应, 实现一步 直接合成 2-氨基咪唑类化合物, 得到中等偏高的产率. 该反应具有很高的应用价值, 可应用于合成具有多种药 物活性的天然 2-氨基咪唑类生物碱 preclathridine A, preclathridine B 和 dorimidazole B. 作者提出了以下反应 机理: 零价钯催化剂由前体 $\mathrm{Pd}(\mathrm{OAc})_{2}$ 还原得到, 接着和 芳基三氟甲磺酸酯发生氧化加成产生离子型钯络合物, 再和炔基进行配位并发生参键反式氨基钯化反应生成 中间体 IM-2, 进行还原消除和双键重排即产生咪唑类 化合物和催化剂, 然后进入新的催化循环.

\section{2 生成胍官能团的反应}

过渡金属催化构建胍官能团反应为合成胍类化合 物的重要方法, 其反应原料相对简单易得, 可高效合成 各种胍类化合物.

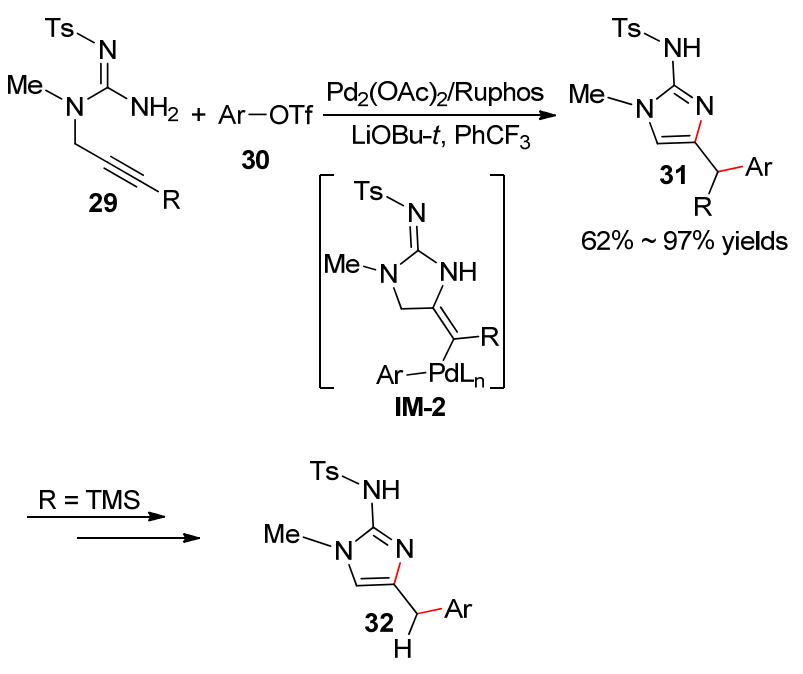

图式 6 钯催化分子内三键胺基化/C- $\mathrm{C}$ 偶联反应合成 2-氨基 咪唑.

Scheme 6 Palladium-catalyzed intramolecular triple bond amination/ $\mathrm{C}-\mathrm{C}$ coupling for the synthesis of 2-aminoimidazoles

\section{1 链状胍类化合物的合成}

多组分反应为一类可以实现一步构建多官能团复 杂化合物的高效合成方法，由于其原料简单、高效、高 选择性和原子经济性的特点, 受到学术界和工业界的青 睐. 最近 Neuville 小组 ${ }^{[28]}$ 发展铜催化氰胺 33、胺 34 和 硼酸 35 的氧化三组分反应高效合成了 $N, N^{\prime}, N^{\prime \prime}$-三取代 胍类化合物 36 的方法(Scheme 7). 通过对反应底物的考 察, 发现一取代脂肪族和芳香族氰胺都很好地反应生成 胍类化合物. 适合于该反应的胺类化合物主要是脂肪 胺，而芳香胺在该氧化反应条件下的反应并没有产物生 成. 含不同取代基的苯基硼酸都适用于该反应，但反应 条件并不能兼容杂环硼酸和环丙基硼酸等热稳定性差 的硼酸. 通过对反应机理进行详细探索, 作者提出了㲵 胺和硼酸偶联及同步进行的胺亲核加成的反应历程. 该 反应所需原料简单易得，一步反应可以构建两个 $\mathrm{C}-\mathrm{N}$ 键, 产率普遍很高, 是一种高效的胍合成方法; 但反应 需要在氧气氛围下进行, 不能兼容一些易被氧化的底物 或者官能团.

我们发现以二芳基三氟甲磺酸碘盐(37)替代嗍酸作 为芳基化试剂, 在铜催化下和氰胺及胺同样也可以发生 三组分反应直接合成 $N, N^{\prime}, N^{\prime \prime}$-三取代胍类化合物 36 (Eq.

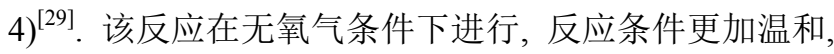
对底物的兼容性好, 易氧化的芳胺如对甲苯胺也可以很 好地进行反应生成目标产物. 芳基硼酸、氧胺和胺的氧 化三组分反应不适合用于合成杂环芳基胍类化合物，而 本反应可应用于对杂环芳基胍的合成. 这很好地弥补了 上述芳基嗍酸参与的三组分反应的不足 ${ }^{[28]}$. 另外, 我们 


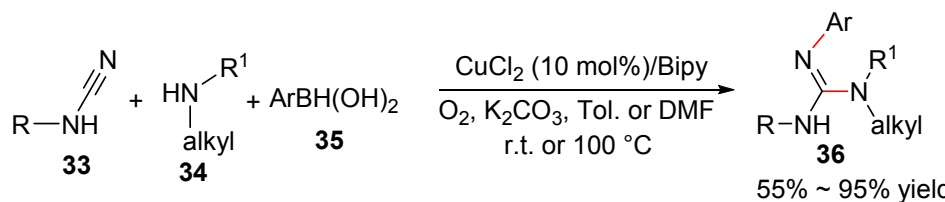

Reaction mechanism:

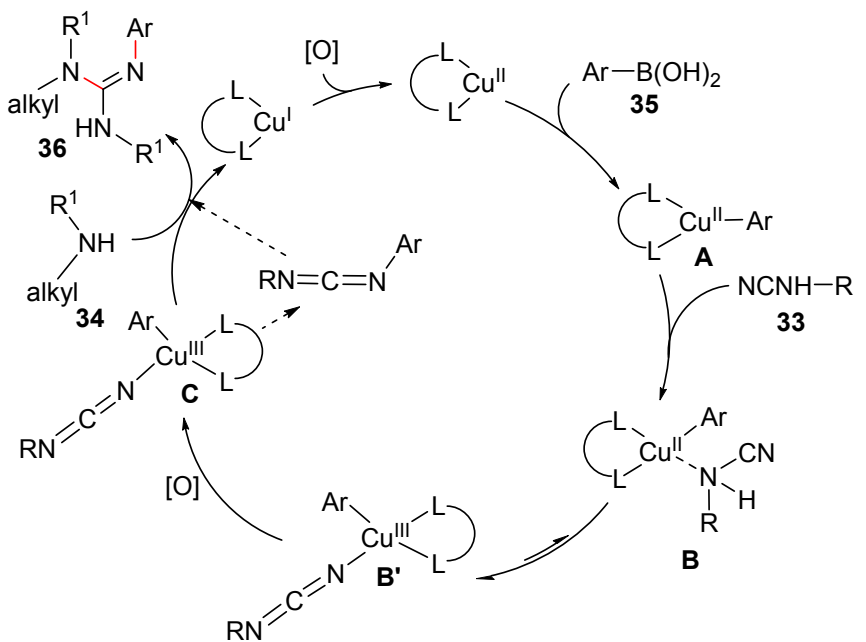

图式 7 铜催化氰胺、胺和嗍酸三组分反应合成胍类化合物

Scheme 7 Copper-catalyzed three-component reaction of cyanamides, amines and boronic acids for the synthesis of guanidines

发现直接活化芳烃的 $\mathrm{C}-\mathrm{H}$ 键, 通过一锅者两步反应也 能够实现对胍类化合物合成, 所用原料简单易得. 由于 二芳基碘盐中只有一个芳基转化为产物, 剩余部分都转 化为副产物, 因此反应的原子利用率很低.

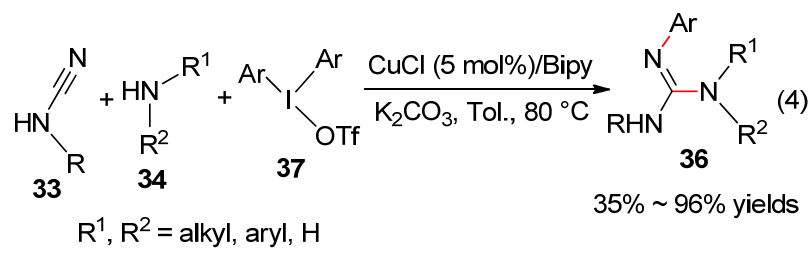

\section{2 环状胍类化合物的合成}

基于分子内 Ullmann 偶联反应, 2011 年 Xi 等 ${ }^{[30]}$ 发 展了 $\mathrm{CuI} / \mathrm{NMP}$ 催化邻卤代苯胺 38 和碳二亚胺 39 的多 米诺反应高效合成了 2-氨基苯并咪唑化合物 $\mathbf{4 0}$ (Eq. 5). 反应经历了以下反应历程: 胺和碳二亚胺的亲核加成生

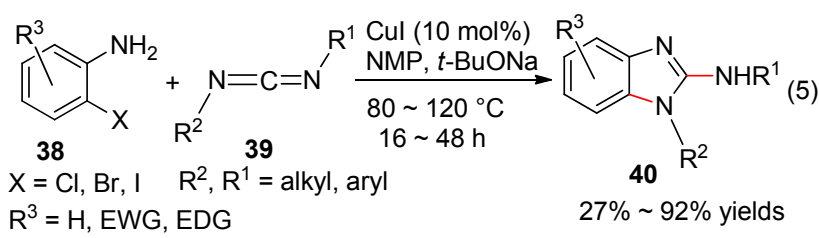<smiles>[R]NC(=NC)Nc1ccc(C)cc1[X]</smiles>

成芳基胍中间体 IM-3，该中间体在铜的催化下接着进 行分子内的 Ullmann 偶联反应生成 2-氨基苯并咪唑. 该 反应可以实现一步构建两个 $\mathrm{C}-\mathrm{N}$ ，且产率普遍很高. 另外, 反应具有较广泛的适用性, 邻碘代苯氨、邻澳苯 氨、邻氯苯氨都适合反应; 反应对不同的官能团如卤素、 酯和硝基都有很好的兼容性.

同样基于 Ullmann 偶联反应, $\mathrm{Ni}$ 等 ${ }^{[31]}$ 最近以二氯 碳亚胺 41 为前体，在铜催化下和两分子胺 $\mathbf{4 2}$ 进行双氨 基化反应生成胍中间体 IM-4，接着发生分子内 $\mathrm{C}-\mathrm{N}$ 偶 联实现对 2-氨基苯并咪唑化合物 43 的合成(Eq. 6) ${ }^{[31]}$. 反应可以一步构建三个 $\mathrm{C}-\mathrm{N}$ 键，高效合成 2-氨基苯并 咪唑, 得到 $38 \% \sim 72 \%$ 的产率.

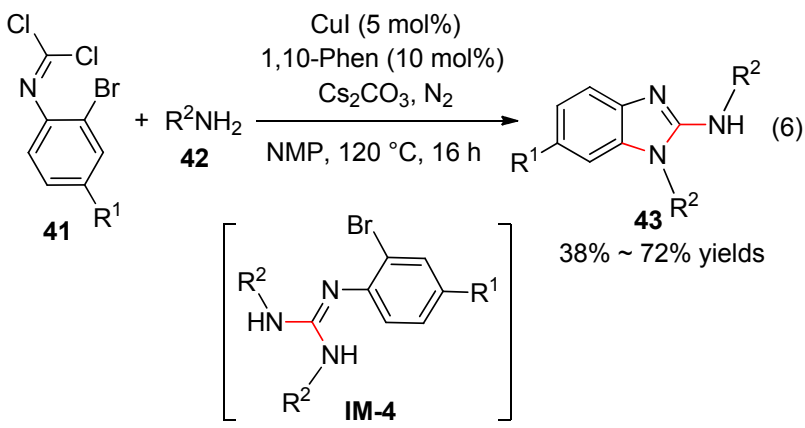

近年来, 随着对 $\mathrm{C}-\mathrm{H}$ 活化反应研究的深入, 过渡 金属催化直接活化 $\mathrm{C}-\mathrm{H}$ 键构筑 $\mathrm{C}-\mathrm{N}$ 键已被成功应用 于合成多种 2-氨基苯并咪唑化合物.

2010 年 Bao 等 ${ }^{[32]}$ 以碳二亚胺 $\mathbf{4 4}$ 和胺 $\mathbf{4 5}$ 为起始原 
料, 发展了铜催化亲核加成和 $\mathrm{C}-\mathrm{H}$ 活化构筑 $\mathrm{C}-\mathrm{N}$ 键 多米诺反应，简便地合成 2-氨基苯并咪唑 46 (Eq. 7).

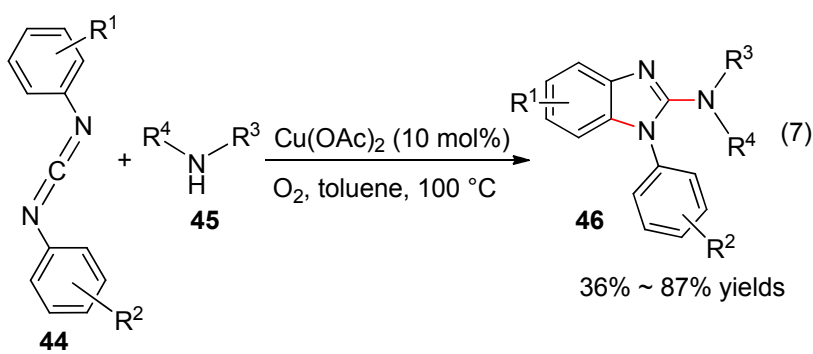

$\mathrm{R}^{1}, \mathrm{R}^{2}=\mathrm{H}$, EWG, EDG $R^{3}, R^{4}=H$, aryl, alkyl

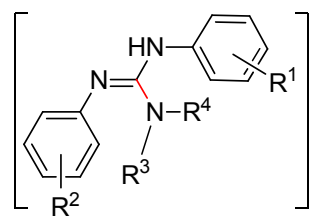

IM-5

在氧化条件下, 碳二亚胺和胺首先进行亲核加成反应生 成胍中间体 IM-5, IM-5 接着发生 C-H 活化/分子内的 $\mathrm{C}-\mathrm{N}$ 偶联反应关环即可生成 2-氨基苯并咪唑化合物. 该反应条件能兼容脂肪族和芳香族胺, 得到中等偏高的 产率. 和基于 Ullmann 反应的合成方法相比 ${ }^{[30]}$, 该方法 的优点为使用原料芳胺简单易得, 不需要先对胍的芳基 进行邻位卤代且反应副产物为无污染的水.

在前期工作的基础上, Neuville 小组 ${ }^{[28]}$ 后来又发展 了铜催化氰胺 47 、胺 48 和芳基硼酸 49 氧化三组分反应 直接合成 2-氨基喹唑啉 50 和 2-氨基苯并咪唑 51 的新方 法(Scheme 8). 在氧气氛围下, $\mathrm{CuCl}_{2} / \mathrm{Bipy}$ 催化氰胺、胺

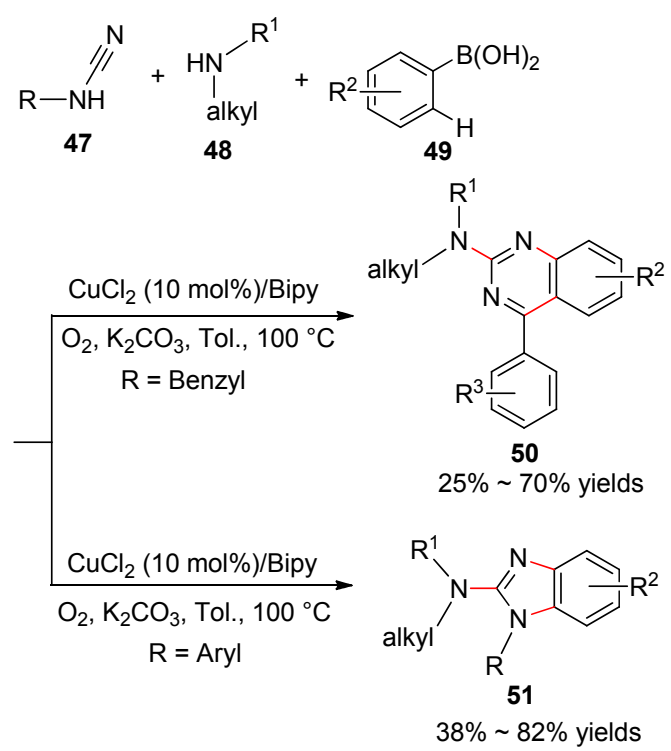

图式 8 铜催化氰胺、胺和硼酸三组分反应合成 2-氨基苯并咪 唑和 2-氨基喹唑啉

Scheme 8 Copper-catalyzed three-component reaction of cyanamides, amines and boronic acids for the synthesis of 2-aminobenz-imidazoles and 2-aminoquinazolines
和芳基嗍酸进行三组分反应，生成中间体 $N, N^{\prime}, N^{\prime \prime}$-三取 代胍, $N, N^{\prime}, N^{\prime \prime}$-三取代胍接着进行分子内的 $\mathrm{C}-\mathrm{H}$ 活化/ $\mathrm{C}-\mathrm{N}$ 偶联反应生成 2-氨基苯并咪唑或者 2-氨基喹唑 啉 ${ }^{[33]}$. 芳基㲵胺参与反应的产物为 2-氨基苯并咪唑，而 苯甲基氰胺参与反应的主要产物为 2-氨基喹唑啉. 该合 成方法原料简单易得，且反应可以实现一步构建三个 $\mathrm{C}-\mathrm{N}$ 键直接合成环状胍类化合物，但产率普遍偏低.

直接活化苯并咪唑 2-位上 $\mathrm{C}$ - $\mathrm{H}$ 键实现分子间氧化 偶联也可以高效合成 2-氨基苯并咪唑. Schreiber 小组 ${ }^{[34]}$ 发现在氧化条件下, $\mathrm{Cu}(\mathrm{OAc})_{2}$ 可以催化酰胺 $\mathbf{5 3}$ 和苯并 咪唑 52 发生分子间的脱氢 $\mathrm{C}-\mathrm{N}$ 偶联生成 2-氨基苯并 咪唑 54 (Eq. 8). 酰胺包括内酰胺、苯甲酰胺和磺酰胺, 都可以和 1-烷基苯并咪唑反应，得到 45\% 97\%的产率. 反应生成副产物为无污染的水，具有高效性和高原子经 济性.

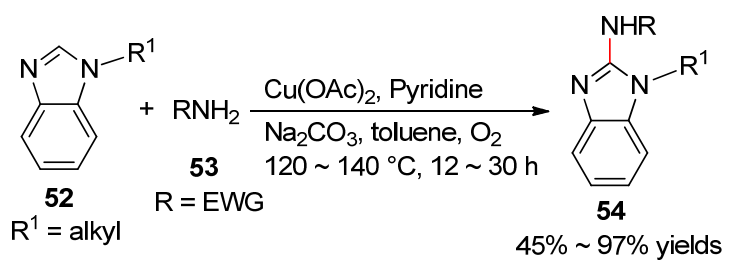

除了上述简单胍类化合物的合成，复杂天然胍生物 碱的合成工作也有了报道. 最近 Wolfe 等 ${ }^{[35]}$ 基于钯催化 烯烃碳胺基化反应，以戊-4-烯醛(55)为起始原料，经过 15 步的反应实现对胍类天然产物 $(+)$-Merobatzelladine $\mathrm{B}$ 的全合成(Scheme 9). 他们 ${ }^{[36]}$ 通过手性辅基诱导的 $\mathrm{Pd}_{2}(\mathrm{dba})_{3}$ 催化不对称烯烃碳氨基化反应构建手性稠环 脲，接着进行亚氨基化和亲核取代反应即可构建三环胍 骨架，再经过多步修饰最终以 $6.7 \%$ 的总收率获得目标 产物 $(+)$-Merobatzelladine B. 后来他们又发展了不对称 $\mathrm{Pd}_{2}(\mathrm{dba})_{3} /(S)-S i p h o s-P E$ 催化烯烃碳氨基化反应构建手 性稠环脲, 并成功应用于合成天然胍生物碱 batzelladine $\mathrm{K}$ 的空间异构体 9-epi-batzelladine $\mathrm{K}^{[37]}$.

\section{3 结论与展望}

胍类化合物因其独特的结构和丰富的生物活性，在 医药、有机合成、农药等领域都有广泛的应用，促进了 胍类化合物合成方法研究的发展. 以胍前体为原料通过 经典的亲核取代和加成反应合成胍的研究已取得重要 的进展，但难以满足各种胍化合物合成的需求. 近年来, 以胍为原料，通过过渡金属催化 $\mathrm{C}-\mathrm{N}$ 生成反应构建较 复杂的非环状或者环状胍化合物的研究也取得可喜的 成果; 然而其合成方法主要基于传统的两组分反应，原 料较为复杂, 需要分离纯化中间体, 且大部分情况下一 次只能构建单个化学键, 以致合成效率较低且能耗高. 

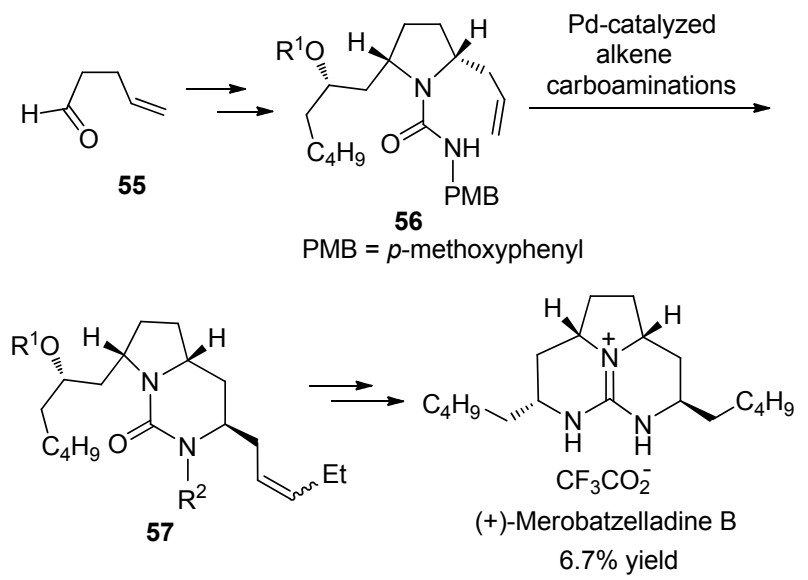

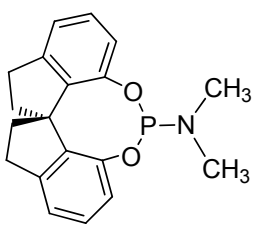

(S)-Siphos-PE

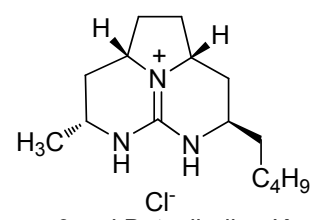

9-epi-Batzelladine $\mathrm{K}$
图式 9 不对称合成天然胍生物碱(+)-Merobatzelladine B 和 9-epi-batzelladine K

Scheme 9 Asymmetric total synthesis of natural (+)-merobatzelladine $\mathrm{B}$ and 9-epi-batzelladine $\mathrm{K}$

另外, 随着对胍类化合物生物活性研究的深入和其在不 同领域更广泛的应用，对胍类化合物的合成也有更高的 要求, 依然面临诸多挑战. 因此, 发展原料简单易得、操 作简便、催化体系高效、适用范围广的绿色合成方法有 很重要的研究意义. 高效的过渡金属催化多组分反应合 成胍将是今后胍类化合物合成研究的一个重要方向. 这 种反应具有操作简单、原料简单易得、不经中间体的分 离、高选择性和高原子经济性等优点, 因此在合成多官 能团复杂的胍类化合物上有很大的优势. 目前这类合成 方法还比较少, 有待于挖掘. 例如, 可基于过渡金属催 化氰胺、胺和硼酸的三组分反应发展新颖的胍类化合物 合成方法, 以实现对多种胍类化合物的高效合成. 通过 利用不同的氰胺、胺或者嗍酸尝试合成官能化的胍中间 体，接着实现胍中间体的环化反应即可构建环状胍. 此 外, 还可以发展不同的过渡金属催化体系, 实现对氧 胺、胺和硼酸的三组分反应选择性的控制, 合成多样性 胍类化合物，拓展反应的应用.

脂肪环状胍是一些复杂天然产物的重要结构单元, 常以稠环结构存在于复杂天然产物中, 这类化合物的合 成方法更具有研究意义和应用价值. 目前这类化合物的 合成方法鲜有报道, 特别稠环化合物的合成方法, 这将 是胍类化合物合成研究的一个研究热点, 也是有待解决 的重点和难点问题.

\section{References}

[1] Tapiero, H.; Mathé, G.; Couvreur, P.; Tew, K. D. Biomed. Pharmacother. 2002, 56, 439.

[2] (a) Heys, L.; Moore, C. G.; Murphy, P. J. Chem. Soc. Rev. 2000, 29, 57.

(b) Urban, S.; Leone, P. A.; Carroll, A. R.; Fechner, G. A.; Smith, J.; Hooper, J. N. A.; Quinn, R. J. J. Org. Chem. 1999, 64, 731.

(c) Hensler, M. E.; Bernstein, G.; Nizeta, V.; Nefzi, A. Bioorg. Med. Chem. Lett. 2006, 16, 5073.

(d) Masuda, T.; Shibuya, S.; Arai, M.; Yoshida, S.; Tomozawa, T.; Ohno, A.; Yamashitab, M.; Honda, T. Bioorg. Med. Chem. Lett. 2003, 13, 669.

[3] Selig, P. Synthesis 2013, 45, 703.

[4] Karmel, I. S. R.; Fridman, N.; Tamm, M.; Eisen, M. S. J. Am. Chem. Soc. 2014, 136, 17180.

[5] (a) Cao, Y.-J.; Gao, H.-X.; Lu, R.-H. Chin. J. Synth. Chem. 2003, 11,193 (in Chinese).

(曹玉娟，高海翔，鲁润华，合成化学, 2003, 11, 19.)

(b) Shen, Z.-X.; Wang, Y.-L.; Zhang, Y.-W. Chin. J. Org. Chem. 2002, 22, 388 (in Chinese).

(沈宗旋, 王亚玲, 张雅文, 有机化学, 2002, 22, 388.)

(c) Ma, Y.; De, S.; Chen, C. Tetrahedron 2015, 71, 1145.

[6] (a) Ni, C.; Zhu, L.; Hu, J. Acta Chem. Sinica 2015, 73, 90 (in Chinese).

(倪传法, 朱林桂, 胡金波, 化学学报, 2015, 73, 90.)

(b) Zhang, W.; Dai, J.; Xu, H. Chin. J. Org. Chem. 2015, 35, 1820 (in Chinese).

(张文曼, 戴建军, 许华建, 有机化学, 2015, 35, 1820.)

[7] Lu, B.; Li, X.; Lin, Y. Chin. J. Org. Chem. 2015, 35, 2275 (in Chinese).

(卢贝丽, 李现艳, 林咏梅, 有机化学, 2015, 35, 2275.)

[8] Tan, M.; Gu, Y.; Luo, X.; Zhang, P. Chin. J. Org. Chem. 2015, 35, 781 (in Chinese)

(谭明雄，顾运琼，罗旭健，张培，有机化学, 2015, 35, 781.)

[9] Qin, Y.; Peng, Q. Chin. J. Org. Chem. 2011, 31,1169 (in Chinese). (秦元成, 彭强, 有机化学, 2011, 31, 1169.)

[10] (a) Xie, J.; Zhou, Q. Acta Chem. Sinica 2012, 70, 1427 (in Chinese).

(谢建华, 周其林, 化学学报, 2012, 70, 1427.)

(b) Zhao, Y.; Guo, C.; Wu, H. Prog. Chem. 2014, 26, 345 (in Chinese).

(赵艳，郭彩红，武海顺，化学进展，2014, 26, 345.)

[11] (a) Zhuo, Z.; Wang, T.; Zhou, X.; Zhang, H. Chin. J. Org. Chem. 2014, 34, 1471 (in Chinese).

(卓庆德，王铜道，周小茜，张弘，有机化学, 2014, 34, 1471.)

(b) Liao, G.; Shi, B. Acta Chim. Sinica 2015, 73, 1283 (in Chinese). (廖港, 史炳锋, 化学学报, 2015, 73, 1283.)

[12] Cortes-Salva, M.; Nguyen, B.-L.; Cuevas, J.; Pennypacker, K. R.; Antilla, J. C. Org. Lett. 2010, 12, 1316.

[13] Xing, H.; Zhang, Y.; Lai, Y.; Jiang, Y.; Ma, D. J. Org. Chem. 2012, $77,5449$.

[14] Miyabe, H.; Yoshida, K.; Reddy, V. K.; Takemoto, Y. J. Org. Chem. 2009, 74, 305.

[15] (a) Zhong, M.; Bui, M.; Shen, W.; Baskaran, S.; Allen, D. A.; Elling, R. A.; Flanagan, W. M.; Fung, A. D.; Hanan, E. J.; Harris, S. O.; Heumann, S. A.; Hoch, U.; Ivy, S. N.; Jacobs, J. W.; Lam, S.; Lee, H.; McDowell, R. S.; Oslob, J. D.; Purkey, H. E.; Romanowski, M. J.; Silverman, J. A.; Tangonan, B. T.; Taverna, P.; Yang, W.; Yoburn, J. C.; Yu, C. H.; Zimmerman, K. M.; O'Brien, T.; Lew, W. Biol. Med. Chem. Lett. 2009, 19, 5158.

(b) Moriya, M.; Kishino, H.; Sakuraba, S.; Sakamoto, T.; Suga, T.; 
Takahashi, H.; Suzuki, T.; Ito, M.; Ito, J.; Moriya, R.; Takenaga, N.; Iwaasa, H.; Ishihara, A.; Kanatani, A.; Fukami, T. Biol. Med. Chem. Lett. 2009, 19, 3568.

(c) Lo, H. Y.; Bentzien, J.; Fleck, R. W.; Pullen, S. S. Biol. Med. Chem. Lett. 2008, 18, 6218.

(d) Xuan, G.; Han, J.; Li X. Eur. J. Med. Chem. 2006, 41, 1080.

(e) Pérez-Villanueva, J.; Hernández-Campos, A.; Yépez-Mulia, L. Biol. Med. Chem. 2013, 21, 4221.

(f) Chen, J.; Gao, L.-X.; Gong, J.-X.; Jiang, C.-S.; Yao, L.-G.; Li, J.-Y.; Li, J.; Xiao, W.; Guo, Y.-W. Biol. Med. Chem. 2015, 23, 2211.

[16] (a) Ueda, S.; Buchwald, S. L. Angew. Chem., Int. Ed. 2012, 51, 10364.

(b) Almași, D.; Alonso, D. A.; Gómez-Bengoa, E.; Nájera, C. J. Org. Chem. 2009, 74, 6163.

(c) Zhang, L.; Lee, M.-M.; Lee, S.-M.; Lee J.; Cheng, M.; Jeong, B.-S.; Park, H.-G.; Jew, S.-S. Adv. Synth. Catal. 2009, 351, 3063.

(d) Gómez-Torres, E.; Alonso, D. A.; Gómez-Bengoa, E.; Nájera, C. Org. Lett. 2011, 13, 6106.

[17] (a) Rastogi, R.; Sharma, S. Synthesis 1983, 861.

(b) Cee, V. J.; Downing, N. S. Tetrahedron Lett. 2006, 47, 3747.

(c) Xie, Y.; Zhang, F.; Li, J.; Shi, X. Synlett 2010, 901.

(d) Deasy, R. E.; Slattery, C. N.; Maguire, A. R.; Kjell, D. P.; Hawk, M. K. N.; Joo, J. M.; Gu, R. L.; Moynihan, H. J. Org. Chem. 2014, 45, 3688 .

(e) Artman, G. D. III; Solovay, C. F.; Adams, C. M.; Diaz, B.; Dimitroff, M.; Ehara, T.; Gu, D.; Ma, F.; Liu, D.; Miller, B. R.; Pick, T. E.; Poon, D. J.; Ryckman, D.; Siesel, D. A.; Stillwell, B. S.; Swiftney, T.; Dyck, J. P.; Zhang, C.; Ji, N. Tetrahedron Lett. 2010, 51,5319

(f) Pollock, J. A.; Kim, S. H.; Katzenellenbogen, J. A. Tetrahedron Lett. 2015, 56, 6097.

[18] Evindar, G.; Batey, R. A. Org. Lett. 2003, 5, 133.

[19] Chi, Y.; Zhang, W.-X.; Xi, Z. Org. Lett. 2014, 16, 6274.

[20] Ueda, S.; Buchwald, S. L. Angew. Chem., Int. Ed. 2012, 51, 10364.

[21] Kim, M.; Mulcahy, J. V.; Espino, C. G.; Bois, J. D. Org. Lett. 2006,

\section{$8,1073$.}

[22] Muñiz, K.; Martínez, C. J. Org. Chem. 2013, 78, 2168.

[23] (a) Schultz, D. M.; Wolfe, J. P. Synthesis (Stuttg) 2012, 44, 351.

(b) Wolfe, J. P. Synlett 2008, 2913.

(c) Wolfe, J. P. Synlett 2006, 571.

[24] Gainer, M. J.; Bennett, N. R.; Takahashi, Y.; Looper, R. E. Angew. Chem., Int. Ed. 2011, 50, 684.

[25] Hövelmann, C. H.; Streuff, J.; Brelot, L.; Muñiz, K. Chem. Commun. 2008, 28, 2334.

[26] Zavesky, B. P.; Babij, N. R.; Fritz, J. A.; Wolfe, J. P. Org. Lett. 2013, 15, 5420 .

[27] Zavesky, B. P.; Babij, N. R.; Wolfe, J. P. Org. Lett. 2014, 16, 4952.

[28] Li, J.; Neuville, L. Org. Lett. 2013, 15, 6124.

[29] Li, J.; Wang, H.; Hou, Y.; Yu, W.; Xu, S.; Zhang, Y. Eur. J. Org. Chem. 2016, 2388.

[30] Wang, F.; Cai, S.; Liao, Q.; Xi, C. J. Org. Chem. 2011, 76, 3174

[31] Yu, H.; Liu, Q.; Li, Y.; Ni, C. Tetrahedron Lett. 2012, 53, 5253.

[32] He, H.-F.; Wang, Z.-J.; Bao, W. Adv. Synth. Catal. 2010, 352, 2905.

[33] Tran, L. Q.; Li, J.; Neuville, L. J. Org. Chem. 2015, 80, 6102.

[34] Wang, Q.; Schreiber, S. L. Org. Lett. 2009, 11, 5178.

[35] (a) Cohen, F.; Overman, L. E. J. Am. Chem. Soc. 2001, 123, 10782. (b) Arnold, M. A.; Day, K. A.; Duron, S. G.; Gin, D. Y. J. Am. Chem. Soc. 2006, 128, 13255.

(c) Evans, P. A.; Qin, J.; Robinson, J. E.; Bazin, B. Angew. Chem., Int. Ed. 2007, 46, 7417.

(d) Ishiwata, T.; Hino, T.; Koshino, H.; Hashimoto, Y.; Nakata, T.; Nagasawa, K. Org. Lett. 2002, 4, 2921.

(e) Rodriguez, R. A.; Steed, D. B.; Kawamata, Y.; Su, S.; Smith, T. C.; Steed, P. A.; Romesberg, F. E.; Baran, P. S. J. Am. Chem. Soc. 2014, 136, 15403.

(f) Ma, Z.; Wang, X.; Ma, Y.; Chen, C. Angew. Chem., Int. Ed. 2016, 55, 4763.

[36] Babij, N. R.; Wolfe, J. P. Angew. Chem., Int. Ed. 2012, 51, 4128.

[37] Babij, N. R.; Wolfe, J. P. Angew. Chem., Int. Ed. 2013, 52, 9247. 